\title{
Association of the ENPPI/ENTPDI Polymorphisms in Hemodialysis Patients
}

\author{
Xi Zhang' \\ Ziming Wan' \\ Si Cheng ${ }^{2}$ \\ Hua Gan' \\ 'Department of Nephrology, The First \\ Affiliated Hospital of Chongqing Medical \\ University, Chongqing, People's Republic \\ of China; ${ }^{2}$ Department of Orthopaedics, \\ The Second Affiliated Hospital of \\ Chongqing Medical University, \\ Chongqing, People's Republic of China
}

Correspondence: Hua Gan

Department of Nephrology, The First Affiliated Hospital of Chongqing Medical University, Chongqing, People's Republic of China

Email correspondzx@।26.com
Introduction: ENPP1 and ENTPD1 are two main enzymes involved in ATP-AMP-ADPadenosine axis, which is associated with lipid metabolism, diabetes mellitus (DM) and renal fibrosis. The single nucleotide polymorphisms (SNPs) of ENPP1 and ENTPD1, rs1044498 and rs6584026, are associated with these factors. This retrospective study aimed to address the two SNPs variants in hemodialysis (HD) patients and analyzes their relations with clinical characteristics.

Methods: This study included 543 regular HD patients over 3 months at our center. Overnight fasting peripheral blood sample was taken from each subject to extract the DNA. The genotypes of rs 1044498 and rs6584026 were detected by polymerase chain reaction-restriction fragment length polymorphism (PCR-RFLP) method. The basic clinical data were noted such as sex, age, and HD-age, and the main causes of chronic kidney disease (CKD) and the clinical characteristics were collected on average at least three times in half a year. $T$-test and Chi-test were performed for the statistical analyses. Binary logistic regression was applied for the significant parameters by excluding the confounders, gender, age and HD-age. All statistical tests were considered significant for $P<0.05$.

Results: The rs1044498 genotypes showed in two types, $\mathrm{A} / \mathrm{A}$ and $\mathrm{A} / \mathrm{C}$ without $\mathrm{C} / \mathrm{C}$. The rs6584026 genotypes were $\mathrm{C} / \mathrm{C}$ and $\mathrm{C} / \mathrm{T}$ without $\mathrm{T} / \mathrm{T}$. The genotype frequency of rs1044498 (A/ C) was 0.238 , and the genotype frequency of $\operatorname{rs} 6584026(\mathrm{C} / \mathrm{T})$ was 0.328 . The age and the level of lipoprotein $\alpha$ showed statistical significance with rs 1044498 variant $(\mathrm{A} / \mathrm{C}, P<0.05)$. The rs6584026 variant $(\mathrm{C} / \mathrm{T})$ was frequently found in patients with nephritis $(P<0.05)$. The albumin, alkaline phosphatase (ALP), lipoprotein $\alpha$, cholesterol, apolipoprotein B (Apo B), Apo B/A1 and nephritis were independently associated with rs6584026 variant $(\mathrm{C} / \mathrm{T}, P<0.05)$ in binary logistic regression model by controlling the confounders of gender, age and HD-age. High level of triglyceride and low level of urine nitrogen were related to rs6584026 variant $(\mathrm{C} / \mathrm{T}, P<0.05)$.

Conclusion: The rs 1044498 and rs6584026 SNPs were related to several high levels of lipids, and rs6584026 variant was related to nephritis and autoimmune disease. The rs6584026 SNP may contribute to the increased risks of cholesterol and ApoB/A1 in HD patients.

Keywords: chronic kidney disease, CKD, single nucleotide polymorphisms, SNPs, ectonucleotide pyrophosphatase/phosphodiesterase 1, ENPP1, ectonucleoside triphosphate diphosphohydrolase 1, ENTPD1, rs1044498, rs6584026

\section{Introduction}

The ATP-ADP-AMP-adenosine axis (Figure 1$)^{1}$ is an important energy metabolism pathway identified in vivo. The enzymes involved in the axis, including NT5E/CD73 (5'-nucleotidase, ecto), ENPP1 (Ectonucleotide pyrophosphatase/phosphodiesterase 1), and ENTPD1/CD39 (Ectonucleoside triphosphate diphosphohydrolase 1) appear to have an association with severe ectopic calcification, ${ }^{2-4}$ which was a common complication on 


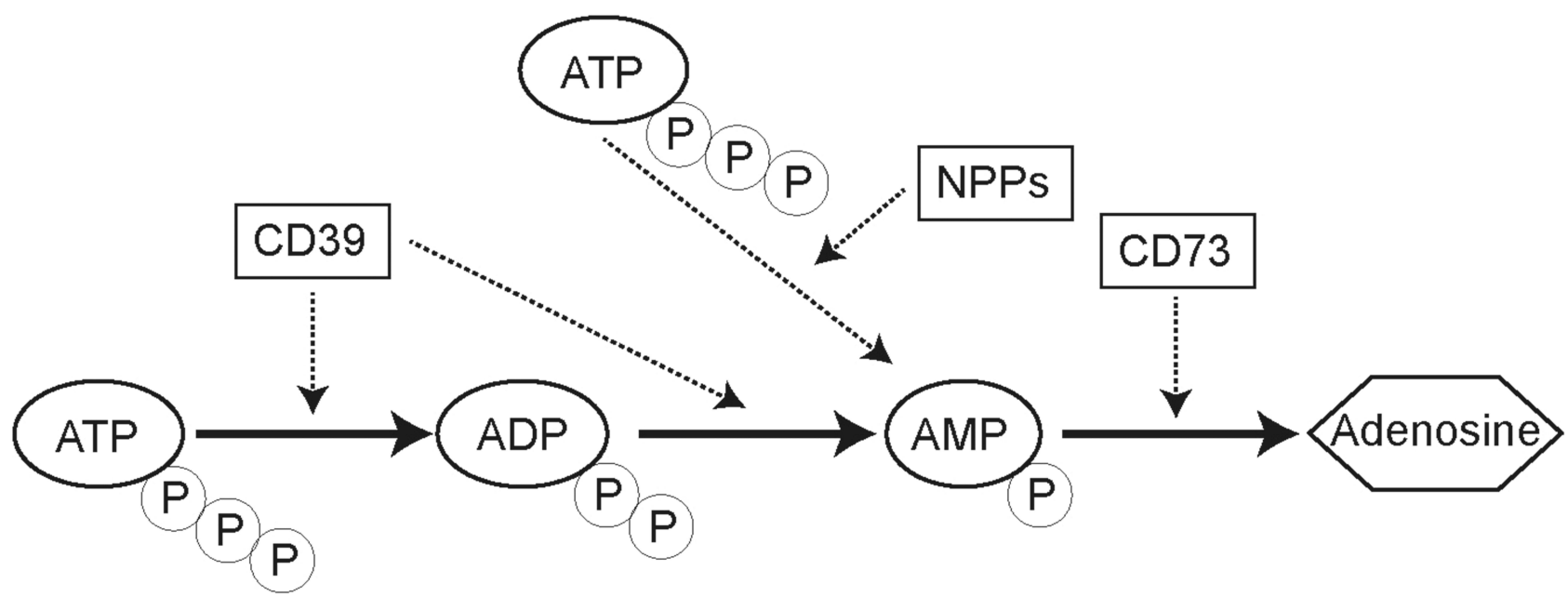

Figure I ATP-ADP-AMP-adenosine axis.

hemodialysis (HD) patients and also tightly connected with cardiovascular disease (CVD).

Besides ectopic calcification, these enzymes were also found to be connected with diabetes mellitus (DM), ${ }^{5}$ abnormal lipid metabolism and ${ }^{6}$ insulin resistance (IR), ${ }^{7}$ which were all related to CVD and chronic kidney disease (CKD).

ENPP1 is a type II transmembrane glycoprotein with extracellular pyrophosphatase and phosphodiesterase activities. ${ }^{8}$ It converts ATP to AMP and pyrophosphate (PPi) outside the cells. ${ }^{9}$ Human ENPP1 gene is 80kb-length and located on chromosome 6q22-q23, consisting of 25 exons and 24 introns, and encoding a 925 -amino acid protein weighted $104.9 \mathrm{kDa}^{10}$ An SNP (Single Nucleotide Polymorphisms) in ENPP1, rs1044498, also known as $\mathrm{K} 121 \mathrm{Q}$, has been found to be related to IR, ${ }^{11}$ type 2 diabetes mellitus (T2DM), ${ }^{5,11,12}$ and coronary heart disease. ${ }^{8}$ Patients with ENPP1 K121Q genotype had a significantly higher coronary calcium score in end-stage renal failure. ${ }^{13}$ The ENPP1 K121Q polymorphism is an independent predictor of major cardiovascular events in high-risk individuals. ${ }^{14}$ These were tightly connected to both CVD and CKD, and CVD was still the leading cause of death in CKD. ${ }^{15-17}$ Most of the studies mentioned above about rs1044498 were mainly focused on Caucasians and African-Americans. In our country, ENPP1 SNP was mainly on T2DM, and the rs1044498 (K121Q) was predisposed to T2DM. ${ }^{18}$ The rs1044498 (K121Q) variant increased susceptibility of DKD in European and Asian population. ${ }^{19}$ Studies on the end stage of CKD in our country are rare, especially in HD patients.
ENTPD1, also known as CD39, is an integral membrane protein. It hydrolyzes ATP and ADP to AMP, and the cascading leads to the generation of adenosine. ${ }^{5}$ Human ENTPD1 is located at chromosome 10, encoding 510 amino acids weighted $57 \mathrm{kDa} .{ }^{20}$ ENTPD1 is reportedly as one of the protective factors against diabetic nephropathy ${ }^{21}$ and related with vascular damage ${ }^{22}$ and inflammation $^{23}$ or tumor development. ${ }^{24}$ One of the most frequently studied SNP in ENTPD1 was rs6584026, and it was mainly restricted to diabetic/non-diabetic condition in an individual and African-American people with CKD. ${ }^{5}$ It lacks rs6584026 variant in HD patients in Chinese.

This hypothesis suggests that there might be some connections between ENPP1 and ENTPD1 in HD patients. Because studies found that the two genes both had tight connections with ectopic calcification, DM and IR et al. And these diseases were also frequently happened in CKD and CVD patients. CVD was still the leading cause of death in CKD, especially in HD patients. We presumed that ENPP1 and ENTPD1 showed significantly differences with some lab data in HD patients. To explore the rs 1044498 and rs6584026 variants in HD patients, we screened the genotypes of the two SNPs in HD patients at a single center and to analyze the relationship between these gene variants and the common lab data.

\section{Materials and Methods}

The study subjects were included from October 2018 to October 2019 at the HD center of the First Affiliated Hospital of Chongqing Medical University. The included criteria: age over 18-year old, diagnosed CKD5 that was 
the $\mathrm{GFR}<15 \mathrm{~mL} / \mathrm{min} / 1.73 \mathrm{~m}^{2}$ AND have already undergoing regular HD for more than 3 months. As the peripheral blood sample was collected before HD, the lipid test must have been taken for at least 3 times in 6 months at our hospital. The excluded criteria: HD-age was less than three months or on peritoneal dialysis, unwilling to take the peripheral blood sample, patient history was not clear or not completed, some clinical data were not enough or/ and missing, the sample was contaminated. The research finally involved 543 subjects. The study was approved by the ethics committee of the First Affiliated Hospital of Chongqing Medical University. Written informed consent was obtained from all the patients.

\section{Clinical Data}

Peripheral blood samples of all subjects were examined after an overnight fasting period. Patient data such as sex (male/female), age (year), HD-age (year), and the main cause of CKD were noted. The main causes of CKD were diagnosed based on the patient history, especially in DM and hypertension (also known as high blood pressure, HBP). Some subjects were both with DM and HBP, the causes of CKD were determined by the primary kidney type-B ultrasound results when they were diagnosed CKD. Normal kidneys construction subjects were classified to the DM, and the symmetrical contracted kidneys were classified to the HBP. The rest were based on the results of renal biopsies in the past, such as ANCA-associated glomerulonephritis (ANCA-GN), hepatitis B virus associated glomerulonephritis (HBV-GN), interstitial nephritis (IN), nephritis (NS) and systemic lupus erythematosus (SLE). Polycystic kidney (PK) was diagnosed by kidney ultrasound or/and abdominal CT scan. In addition, the clinical parameters were collected on average at least three times in 6 months. Clinical data included serum creatinine, urine nitrogen, albumin, globulin, hemoglobin, calcium, phosphate, alkaline phosphatase (ALP), parathyroid hormone, triglyceride, cholesterol, low-density lipoprotein (LDL), high-density lipoprotein (HDL), lipoprotein $\alpha$, apolipoprotein A1 (Apo A1), apolipoprotein $\beta$ (Apo B), and Apo B/A1. All the clinical lab data were retrieved from patients' case sheets.

\section{Genotyping}

Genomic DNA was extracted and purified from peripheral blood using a salting-out procedure. $1 \mathrm{~mL}$ peripheral blood samples were collected by EDTA- $\mathrm{K}_{2}$ anticoagulated tubes. Added 2-3 times volumes of blood buffer, vortex mixed
15 seconds, then centrifuged (8000r/min, $8 \mathrm{~min})$, discarded the supernatant. Repeated this step 2-3 times. Added $0.3 \mathrm{~mL} \mathrm{SE}$ solutions and mixed by vortex. Cell lysates were digested overnight at $37^{\circ} \mathrm{C}$ with proteinase $\mathrm{K}$ and $10 \%$ sodium dodecyl sulphate. $6 \mathrm{M} \mathrm{NaCl}$ and nucleic lysis buffer were added, and then centrifuged (2000r/min, $15 \mathrm{~min})$. DNA was precipitated by adding $96 \%$ ethanol. DNA strands were removed by sterile glass rod to a new tube, washed by $70 \%$ ethanol and left to dry out. DNA was added with TE buffer and allowed to dissolve $24 \mathrm{~h}$. The products used were from a DNA Isolation-kit (Sangon, Shanghai, China). The extracted DNA was evaluated by measuring its concentration and purity spectrophotometrically using a NanoDrop 1000 Spectrophotometer. The ratio of the absorbance at $260 \mathrm{~nm}$ and $280 \mathrm{~nm}$ was used to define DNA purity. The value in the range $1.8-2.0$ was considered to represent pure DNA as it is in good deproteinization.

The ENPP1 and ENTPD1/CD39 polymorphisms were detected using the polymerase chain reaction-restriction fragment length polymorphism (PCR-RFLP) method. Sequences of the primer pairs used were 5'GTTGCTCATGGATCATACTCAGG-3' (forward primer) and 5'-TATGAAGCTGGGTGATGGGTAT-3' (reverse primer) for ENPP1 and 5'-GAAGCAACCTAAGTGTCCG TCA-3' (forward primer) and 5'-CAATGACAGGGAGAA GGGAAA-3' (reverse primer) for ENTPD1. PCR was performed using $1 \mu \mathrm{L}$ genomic DNA in a total volume of $25 \mu \mathrm{L}$ reaction that included $10 \mathrm{X}$ PCR buffer, $25 \mathrm{mM}$ $\mathrm{MgCl}_{2}, 0.5 \mu \mathrm{L}$ of each primer, $0.5 \mu \mathrm{L}$ of $10 \mathrm{mM} \mathrm{dNTPs}$, and 5U Taq polymerase. The products used were from a PCR-kit (Sangon, Shanghai, China). PCR conditions were as follows: initial denaturation at $95^{\circ} \mathrm{C}$ for $3 \mathrm{~min}$, followed by 35 cycles of denaturation at $94^{\circ} \mathrm{C}$ for $30 \mathrm{~s}$, annealing at $58^{\circ} \mathrm{C}$ for $30 \mathrm{~s}$, and extension at $72^{\circ} \mathrm{C}$ for $30-$ $60 \mathrm{~s}$, with a final extension at $72^{\circ} \mathrm{C}$ for $10 \mathrm{~min}$. The PCR products were digested for $4 \mathrm{~h}$ at $37^{\circ} \mathrm{C}$ with $3 \mathrm{U}$ AvaII (ThermoFisher, Shanghai, China). The fragments were resolved using 1.5\% agarose gel electrophoresis. The accuracy of genotyping has been further confirmed by direct sequencing of 30 randomly chosen subjects.

\section{Statistical Analysis}

All statistical analyses were performed using SPSS statistics V22.0. Student's $t$-test and the analysis of variance were used to analyze continuous variables and expressed as mean \pm standard deviation. The rank sum test was performed, while the continuous variables were uneven. 
Counts were analyzed using the chi-square test. The associated characteristics were evaluated in a binary logistic regression model. All statistical tests were considered significant for $P<0.05$.

\section{Results}

The study involved 1022 subjects randomly searched by hospital administrator software. It excluded out HD-age for less than 3 months or undergoing peritoneal dialysis $(n=97)$, clinical data were not enough or missing $(n=332)$, patient history was not clear or not completed $(\mathrm{n}=36)$, unwilling to take the peripheral blood sample at last $(\mathrm{n}=13)$, and contaminated blood sample $(\mathrm{n}=1)$ (Figure 2). The clinical data meant most did not have at least three times blood tests at our hospital in 6 months or the data were untraceable.

The clinical characteristics of the subjects with different genotypes are summarized in Table 1. All the subjects genotypes showed in two different types, $\mathrm{A} / \mathrm{A}$ and $\mathrm{A} / \mathrm{C}$ without $\mathrm{C} / \mathrm{C}$ in $\mathrm{rs} 1044498, \mathrm{C} / \mathrm{C}$ and $\mathrm{C} / \mathrm{T}$ without $\mathrm{T} / \mathrm{T}$ in rs6584026. The rs 1044498 variant type was $\mathrm{A} / \mathrm{C}$, and the rs6584026 variant type was $\mathrm{C} / \mathrm{T}$. The rs 1044498 variant (A/C) was statistically significant when compared to the age and the level of lipoprotein $\alpha$ (Table $1, P<0.05$ ). The

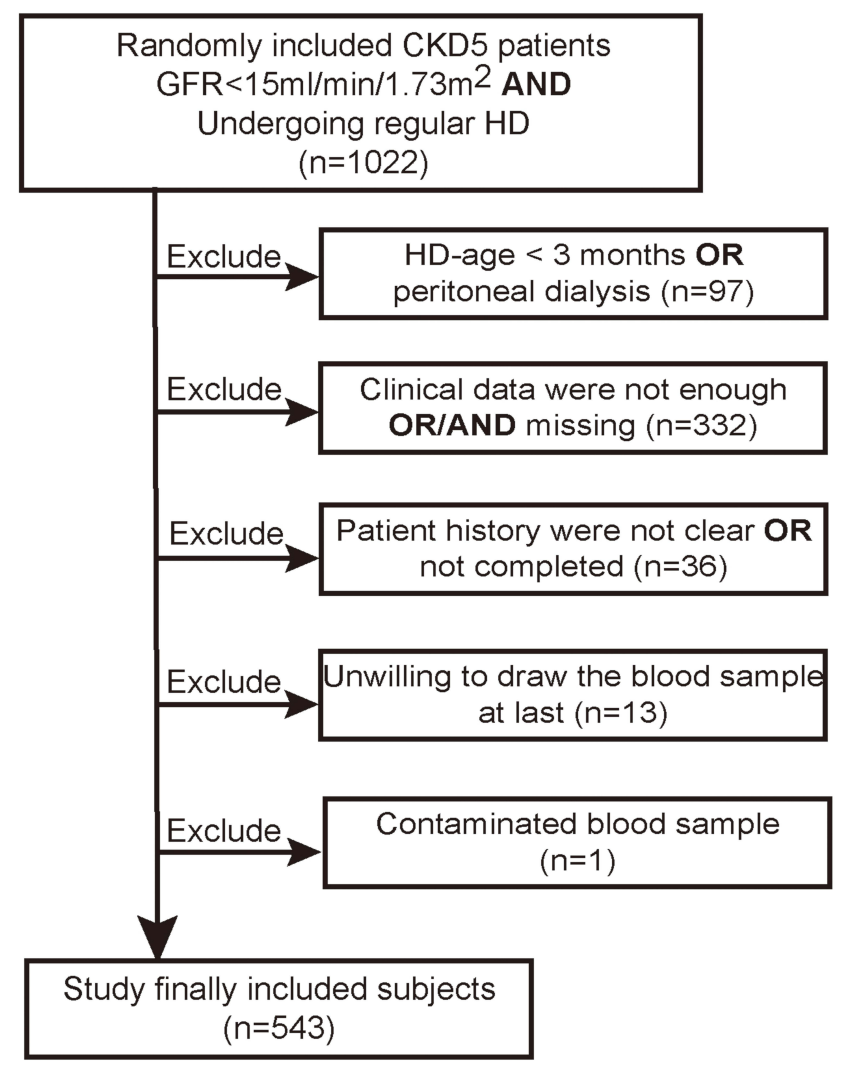

Figure 2 The flow chart of subjects included and exclude. rs6584026 variant $(\mathrm{C} / \mathrm{T})$ was found to be associated with high levels of urine nitrogen, albumin, ALP, lipoprotein $\alpha$, cholesterol, triglyceride, Apo B, and Apo B/A1 (Table 1, $P<0.05)$. No significant correlation was found between the rest of the clinical parameters and variants of rs1044498 $(\mathrm{A} / \mathrm{C})$ and $\mathrm{rs} 6584026(\mathrm{C} / \mathrm{T})(\mathrm{p}>0.05)$. Further, no statistically significant association was observed between the SNP variants and the patient gender ( $p>0.05)$.

There were eight cause diseases to $\mathrm{CKD}$, and they were classified into DM and non-DM, HBP and non-HBP, autoimmune diseases (AD) and non-AD shows in Table 2. The AD included SLE and ANCA-GN. While no significant correlation was found between these diseases and the rs1044498 (A/C) variant ( $>>0.05)$. The $\operatorname{rs6584026}(\mathrm{C} / \mathrm{T})$ variant was found to be significantly associated with NS and AD, including ANCA and SLE (Table 2, $P<0.05$ ) but not with DM or HBP (Table 2, $P>0.05$ ). According to these subjects, the rs $1044498 \mathrm{~A} / \mathrm{C}$ genotype frequency was 0.238 , and the $\mathrm{rs} 6584026 \mathrm{C} / \mathrm{T}$ genotype frequency was 0.328 .

Binary logistic regression model was applied to estimate the association between rs6584026 genotype and some factors, including urine nitrogen, albumin, ALP, lipoprotein $\alpha$, cholesterol, triglyceride, ApoB/A1 and NS with non-NS, by excluding confounding effects like age, gender and HD-age. The results revealed that albumin, ALP, lipoprotien $\alpha$, cholesterol, triglyceride, ApoB/A1 and NS were independently associated with the rs6584026 genotype (Table 3).

\section{Discussion}

This retrospective study showed the variants of ENPP1 (rs1044498) and ENTPD1/CD39 (rs6584026) in HD patients in the subjects enrolled from a single center in China. The was a review that rs 1044498 variant in Asia was based on T2DM patients. The frequency of these SNPs variants was not well studied in HD patients, especially in Chinese. In our study, the main findings were the rs6584026 variants $(\mathrm{C} / \mathrm{T})$ were related to high levels of the lipids, lipoprotein $\alpha$, cholesterol, triglyceride, Apo B, and Apo B/A1. There was a report that high-level cholesterol was related to CD39 activity in human beings. ${ }^{25}$ We have not found reference about high level of lipid effected the CD39 expression in HD patients. The variant of rs6584026 was also frequently found in patients with nephritis. Lipid metabolism dysfunction and inflammation were the main complication and pathophysiology in nephritis and autoimmune disease progressing. ${ }^{26,27}$ Lipid metabolic 
Table I The Distributions of DM/Non-DM, HBP/Non-HBP, NS/Non-NS and AD/Non-AD with Different rs $1044498 / \mathrm{rs} 6584026$ Genotypes and Their Genotype Frequencies

\begin{tabular}{|c|c|c|c|c|c|c|c|}
\hline & & \multicolumn{2}{|c|}{ rs 1044498} & \multirow[t]{2}{*}{$P$} & \multicolumn{2}{|c|}{ rs6584026 } & \multirow[t]{2}{*}{$P$} \\
\hline & & A/A & $\mathrm{A} / \mathrm{C}$ & & $\mathrm{C} / \mathrm{C}$ & $\mathrm{C} / \mathrm{T}$ & \\
\hline Age(Year) & $57.08 \pm 15.21$ & $56.49 \pm 15.59$ & $58.95 \pm 13.77$ & 0.031 & $55.99 \pm 15.80$ & $59.32 \pm 13.70$ & 0.063 \\
\hline Sex (Male/Female) & $4 \mid 4 / 129$ & $228 / 186$ & $78 / 51$ & 0.310 & $210 / 156$ & $96 / 81$ & 0.519 \\
\hline HD-age(Year) & $3.97 \pm 3.01$ & $3.99 \pm 3.02$ & $3.91 \pm 3.00$ & 0.777 & $3.83 \pm 2.84$ & $4.26 \pm 3.32$ & 0.369 \\
\hline Calcium & $2.19 \pm 0.28$ & $2.19 \pm 0.28$ & $2.17 \pm 0.27$ & 0.424 & $2.18 \pm 0.29$ & $2.20 \pm 0.25$ & 0.507 \\
\hline Phosphate & $1.69 \pm 0.59$ & $1.71 \pm 0.59$ & $1.63 \pm 0.63$ & 0.187 & $1.71 \pm 0.58$ & $1.66 \pm 0.62$ & 0.411 \\
\hline Serum creatinine & $716.59 \pm 294.13$ & $724.41 \pm 303.33$ & $691.51 \pm 261.33$ & 0.577 & $728.75 \pm 288.68$ & $691.46 \pm 304.00$ & 0.166 \\
\hline Urine nitrogen & $18.94 \pm 9.67$ & $19.20 \pm 9.79$ & $18.10 \pm 9.23$ & 0.256 & $19.75 \pm 9.62$ & $17.27 \pm 9.57$ & 0.005 \\
\hline Albumin & $37.71 \pm 5.82$ & $37.73 \pm 6.08$ & $37.65 \pm 4.90$ & 0.620 & $37.20 \pm 5.73$ & $38.76 \pm 5.86$ & 0.003 \\
\hline Globulin & $50.57 \pm 19.61$ & $49.99 \pm 19.61$ & $52.44 \pm 19.54$ & 0.214 & $52.30 \pm 19.05$ & $51.14 \pm 20.74$ & 0.164 \\
\hline Alkaline phosphatase & $156.69 \pm 212.36$ & $155.48 \pm 220.45$ & $160.58 \pm 184.28$ & 0.812 & $143.58 \pm 202.49$ & $183.80 \pm 229.40$ & 0.038 \\
\hline Parathyroid Hormone & $445.82 \pm 563.04$ & $443.92 \pm 594.75$ & $451.91 \pm 446.94$ & 0.888 & $427.41 \pm 551.28$ & $483.88 \pm 585.60$ & 0.273 \\
\hline Hemoglobin & $103.4 \pm 23.67$ & $103.89 \pm 23.34$ & $101.84 \pm 24.69$ & 0.390 & $102.05 \pm 23.71$ & $106.20 \pm 23.36$ & 0.055 \\
\hline High-density lipoprotein & $1.14 \pm 0.39$ & $1.14 \pm 0.37$ & $1.15 \pm 0.45$ & 0.767 & $1.14 \pm 0.37$ & $1.13 \pm 0.42$ & 0.707 \\
\hline Low-density lipoprotein & $2.30 \pm 0.79$ & $2.28 \pm 0.75$ & $2.32 \pm 0.90$ & 0.963 & $2.25 \pm 0.73$ & $2.37 \pm 0.89$ & 0.273 \\
\hline Lipoprotein $\alpha$ & $403.88 \pm 410.06$ & $351.96 \pm 323.21$ & $570.49 \pm 581.03$ & 0.000 & $372.95 \pm 358.14$ & $467.73 \pm 495.21$ & 0.031 \\
\hline Cholesterol & $3.90 \pm 1.14$ & $3.90 \pm 1.02$ & $3.92 \pm 1.49$ & 0.156 & $3.72 \pm 1.04$ & $4.27 \pm 1.26$ & 0.000 \\
\hline Triglyceride & $1.72 \pm 1.35$ & $1.74 \pm 1.26$ & $1.64 \pm 1.61$ & 0.470 & $1.58 \pm 1.21$ & $2.01 \pm 1.57$ & 0.000 \\
\hline ApolipoproteinAI & $1.19 \pm 0.27$ & $1.20 \pm 0.25$ & $1.17 \pm 0.33$ & 0.261 & $1.18 \pm 0.25$ & $1.21 \pm 0.32$ & 0.563 \\
\hline Apolipoprotein B & $0.80 \pm 0.27$ & $0.80 \pm 0.24$ & $0.81 \pm 0.34$ & 0.231 & $0.76 \pm 0.24$ & $0.88 \pm 0.30$ & 0.000 \\
\hline ApoB/Al & $0.70 \pm 0.30$ & $0.69 \pm 0.24$ & $0.76 \pm 0.43$ & 0.806 & $0.67 \pm 0.24$ & $0.78 \pm 0.38$ & 0.000 \\
\hline
\end{tabular}

Notes: Except the data for gender, data are expressed as mean \pm SD. Student's $t$-test and analysis of variance were used to analyze continuous variables. The rank sum test was performed while the continuous variables were uneven. $P<0.05$ shows values are statistical significantly in bold.

dysfunction is significantly associated with CKD, CVD and its mortality. The top death threat is still CVD in CKD patients. ${ }^{28}$ Maybe the rs6584026 SNP took important roles in the pathophysiological process from the beginning to the end stage of renal disease. However, the data were collected at the end stage of renal disease, while the lipid levels and inflammation mostly stayed undercontrolled. The past lipids data were hard to trace. We could not confirm if the time-length or the extent of lipid levels caused the genotype variant. It needed large samples from multiple centers with a long-time follow-up to collected the data. In addition, in the deep analysis between DM and NS groups, we showed that the LDL, lipoprotein $\alpha$ and Apo B levels were higher in DM group than those in NS group (data not show). But in our results, the rs6584026 significant variant is shown in the NS group, not the DM group. It revealed that the rs6584026 variant was related to high level of lipids, but not just limited to the levels. It might be associated with some other reasons, such as inflammations, immune responses, energy metabolism and some signal pathways. ${ }^{29,30}$ In our opinion, high levels of lipids, some inflammations and multiple signal pathways might all together cause the variant of rs6584026.

In nephritis, the main pathophysiology is inflammatory injury in the kidney, which continuously ends with renal fibrosis, following the renal dysfunction. Meanwhile, it has been established that adenosine can inhibit the advancement of renal fibrosis through a combination of A2A and A2B receptors on renal tubular endothelial cells. ${ }^{31}$ Treg 
Table 2 The Clinical Characteristics of Subjects with Different Genotypes (rs I044498/rs6584026)

\begin{tabular}{|c|c|c|c|c|c|c|}
\hline & rs1044498 & & $P$ & rs6584026 & & $P$ \\
\hline & A/A & $A / C$ & & $\mathrm{C} / \mathrm{C}$ & $\mathrm{C} / \mathrm{T}$ & \\
\hline DM & 102 & 36 & 0.488 & 84 & 54 & 0.059 \\
\hline Non-DM & 312 & 93 & & 282 & 123 & \\
\hline HBP & 102 & 27 & 0.410 & 84 & 45 & 0.521 \\
\hline Non-HBP & 312 & 102 & & 282 & 132 & \\
\hline NS & 123 & 39 & 0.913 & 132 & 30 & 0.000 \\
\hline Non-NS & 291 & 90 & & 234 & 147 & \\
\hline$A D$ & 15 & 3 & 0.472 & 18 & 0 & 0.003 \\
\hline Non-AD & 399 & 126 & & 348 & 177 & \\
\hline $\begin{array}{l}\text { Genotype } \\
\text { frequency }\end{array}$ & 414 & 129 & 0.238 & 366 & 177 & 0.328 \\
\hline
\end{tabular}

Notes: Counts were analyzed using the chi-square test. $P<0.05$ shows values are statistical significantly in bold.

Abbreviations: DM, diabetes mellitus; HBP, high blood pressure; NS, nephritis; AD, autoimmune diseases; SLE, systemic lupus erythematosus; ANCA-GN, ANCAassociated glomerulonephritis.

cells or macrophages were involved in renal protection mediated by $\mathrm{CD} 39,{ }^{32,33}$ even though the macrophages were also one of the main inflammatory cells in kidney injury. Overexpression CD39 fails to protect against renal fibrosis. ${ }^{34}$ In these researches, some results were contradicted to each other. In my opinion, this could be explained by the limitations of different subjects, such as humans, ${ }^{29}$ mice or transgenic mice, ${ }^{33}$ or different study models or factors, such as unilateral ureteric obstructive

Table 3 Association of rs6584026 Genotype with Some Characteristics

\begin{tabular}{|l|l|l|}
\hline & \multicolumn{1}{|c|}{ OR(95\% CI) } & $\boldsymbol{P}$ \\
\hline Urine nitrogen & $0.9817(0.960-1.003)$ & 0.278 \\
\hline Albumin & $1.087(1.046-1.130)$ & $\mathbf{0 . 0 0 0}$ \\
\hline Alkaline phosphatase & $1.001(1.000-1.002)$ & $\mathbf{0 . 0 4 3}$ \\
\hline Lipoprotein $\alpha$ & $1.001(1.000-1.001)$ & $\mathbf{0 . 0 4 5}$ \\
\hline Cholesterol & $1.633(1.160-2.300)$ & $\mathbf{0 . 0 0 5}$ \\
\hline Triglyceride & $1.018(0.865-1.198)$ & 0.828 \\
\hline Apo B/AI & $6.275(2.147-18.345)$ & $\mathbf{0 . 0 0 1}$ \\
\hline NS and non-NS & $0.298(0.182-0.49 I)$ & $\mathbf{0 . 0 0 0}$ \\
\hline
\end{tabular}

Notes: The rs6584026 genotype was estimated by binary logistic regression analysis with adjusted confounders, sex, age and HD-age. $P<0.05$ shows values are statistical significantly in bold.

Abbreviations: $\mathrm{OR}$, odds ratio; $\mathrm{Cl}$, confidence interval; NS, nephritis. model $^{34}$ and angiotensin II. ${ }^{35}$ Above all, it was inferred that the adenosine signaling pathway was blocked in presence of rs6584026 mutation leading to an increase in inflammation and subsequent advancement of renal fibrosis. According to our results, NS was a significant independent valuable for rs6584026 variant, but the effect was minor. Until now, it is not clear that SNP variant might consequently come with renal diseases or the diseases caused the SNP variant in humans. In the future study, our group is planning to do an observational study to confirm the logical relations between the renal diseases and the SNP variants.

Besides lipid metabolism and inflammation, the relations among calcification, DM, CKD and CVD mainly focused on ATP-AMP-ADP-adenosine axis. ${ }^{8,36-39}$ All the conditions were tightly connected with CKD itself, not only as part of the cause of disease but as complications, especially the CVD complication, which was still the leading cause of death in CKD5 patients. These enzymes involved in the axis were regulated through lipid metabolic dysfunction and inflammation in the genomic pathway. Cell energy metabolism was different from the change of the enzyme expressions, which conversely changed the lipid metabolism. ${ }^{40}$ The SNPs of ENPP1 and ENTPD1 mainly involved in this ATP-AMP-ADP-adenosine axis are considerably significant in future study because of the tight connections between CVD and CKD. All the 
factors involved were important to the diseases and consequent complications.

Generally, one protein has several SNPs for translation and expression. Initially, nearly all enzymes related to the whole ATP-AMP-ADP-adenosine axis were screened and tested in primary study. However, rs373328681 and rs387906620 (CD73/NT5E) $)^{2,41}$ showed no variant in these subjects. The rs12763743 and rs3897983 (ENTPD1/CD39 $^{5}$ also showed no variant in the preliminary screening. Therefore, the above SNPs were excluded from the remaining studies. Most referenced studies about rs1044498 and rs6584026 revealed their association with abnormal lipid metabolism and kidney diseases, but few studies included CKD patients or HD patients and analyzed the associations between SNP variants and clinical parameters - a potential new angle to be explored in CKD field. How the lipid metabolism dysfunction caused the variant of rs6584026 or change the ENTPD1 expression might be the next study plan in the future.

This study was on the ENPP1/ENPTD1 SNPs variants in hemodialysis patients. It was limited in a single center and hundreds of sample size. Several causes of diseases, such as autoimmune diseases, needed larger sample size to explore the variants of these SNPs. As a result, it was excluded in the binary logistic regression analysis.

In conclusion, the rs1044498 and rs6584026 SNPs were related to several high levels of lipids, and rs6584026 variant was also related to nephritis and autoimmune disease. The rs6584026 SNP may contribute to the increased risk of cholesterol and ApoB/A1 in HD patients.

\section{Acknowledgments}

We sincerely thank LX Chen et al at the central laboratory of the First Affiliated Hospital of Chongqing Medical University for their assistance in conducting our experiments.

\section{Author Contributions}

All authors made a significant contribution to the work reported, whether that is in the conception, study design, execution, acquisition of data, analysis and interpretation, or in all these areas; took part in drafting, revising or critically reviewing the article; gave final approval of the version to be published; have agreed on the journal to which the article has been submitted; and agree to be accountable for all aspects of the work.

\section{Funding}

This research was approved and supported by the Hospital Foundation of the First Affiliated Hospital of Chongqing Medical University.

\section{Disclosure}

The authors declare no conflict of interest. The funders had no role in the study design, the collection, analyses, or interpretation of data, manuscript writing, or in the decision to publish the results.

\section{References}

1. Kishore BK, Robson SC, Dwyer KM. CD39-adenosinergic axis in renal pathophysiology and therapeutics. Purinerg Signal. 2018;14 (2):109-120. doi:10.1007/s11302-017-9596-x

2. Hilaire CS, Ziegler SG, Markello TC, et al. NT5E mutations and arterial calcifications. $N$ Eng $J$ Med. 2011;364(5)::432-42. doi:10.1056/NEJMoa0912923

3. Kochan Z, Karbowska J, Gogga P, et al. Polymorphism in exon 6 of the human NT5E gene is associated with aortic valve calcification. Nucleosides Nucleotides Nucleic Acids. 2016;35(10-12):726-731. doi:10.1080/15257770.2016.1180393

4. Albright RA, Stabach P, Cao WX, et al. ENPP1-Fc prevents mortality and vascular calcifications in rodent model of generalized arterial calcification of infancy. Nat Commun. 2015;1(6):10006. doi:10.10 38/ncomms 10006

5. Friedman DJ, Talbert ME, Bowden DW, et al. Functional ENTPD1 polymorphisms in African Americans with diabetes and end-stage renal disease. Diabetes. 2009;58(4)::999-1006. doi:10.2337/db081214

6. Michos ED, McEvoy JW, Blumenthal RS. Lipid management for the prevention of atherosclerotic cardiovascular disease. $N$ Eng J Med. 2019;381(16):1557-1567. doi:10.1056/NEJMra1806939

7. Wu HZ, Ballantyne CM. Metabolic inflammation and insulin resistance in obesity. Circ Res. 2020;126(11):1549-1564. doi:10.1161/ CIRCRESAHA.119.315896

8. Di JY, Dai ML, Zhang ZX. ENPP1 K121Q (rs1044498C>A) genetic polymorphism confers a high risk of susceptibility to coronary heart disease. Medicine. 2018;97(27):27. doi:10.1097/MD.00000000000 11236

9. Jin YY, Cong Q, Gvozdenovic-Jeremic J, et al. Enpp1 inhibits ectopic joint calcification and maintains articular chondrocytes by repressing Hedgehog signaling. Development. 2018;145(18):dev164830. doi: $10.1242 /$ dev. 164830

10. Abate N, Chandalia M, Di Paola R, et al. Mechanisms of disease: Ectonucleotide pyrophosphatase phosphodiesterase 1 as a gatekeeper of insulin receptors. Nat Clin Pract Endocrinol Metab. 2006;2 (12):694-701. doi:10.1038/ncpendmet0367

11. Bhatti JS, Bhatti GK, Mastana SS, et al. ENPP1/PC-1 K121Q polymorphism and genetic susceptibility to type 2 diabetes in North Indians. Mol Cell Biochem. 2010;345(1-2):249-257. doi:10.1007/ s11010-010-0579-2

12. Chen LL, Qin YF, Liang DY, et al. Gender differences in the association of ENPP1 polymorphisms with type 2 diabetes in a Chinese population. Gene. 2017;30(637):190-195. doi:10.1016/j.gene.201 7.09 .052 
13. Eller P, Hochegger K, Feuchtner GM, et al. Impact of ENPP1 genotype on arterial calcification in patients with end-stage renal failure. Nephrol Dial Transplant. 2008;23(1):321-327. doi:10.1093/ndt/ gfm566

14. Bacci S, Rizza S, Prudente S, et al. The ENPP1 Q121 variant predicts major cardiovascular events in high-risk individuals: evidence for interaction with obesity in diabetic patients. Diabetes. 2011;60 (3):1000-1007. doi:10.2337/db10-1300

15. Bikbov B, Purcell CA, Levey AS; Collaboration GCKD. Global, regional, and national burden of chronic kidney disease, 19902017: a systematic analysis for the Global Burden of Disease Study 2017. Lancet. 2020;395(10225):709-733. doi:10.1016/S0140$6736(20) 30045-3$

16. Zhang LX, Wang F, Wang L, et al. Prevalence of chronic kidney disease in China: a cross-sectional survey. Lancet. 2012;379 (9818):815-822. doi:10.1016/S0140-6736(12)60033-6

17. Huang QX, Li JB, Huang NY, et al. Elevated osteoprotegerin concentration predicts increased risk of cardiovascular mortality in patients with chronic kidney disease: a systematic review and meta-analysis. Kidney Blood Press R. 2020;45(4):565-575. doi:10.1159/000508978

18. Li YY. ENPP1 K121Q polymorphism and type 2 diabetes mellitus in the Chinese population: a meta-analysis including 11855 subjects. Metabolism. 2011;61(5):625-633. doi:10.1016/j.metabol.2011.10.002

19. Sortica DA, Buffon MP, Souza BM, et al. Association between the ENPP1 K121Q polymorphism and risk of diabetic kidney disease: a systematic review and meta-analysis. PLoS One. 2015;10(3): e0118416. doi:10.1371/journal.pone.0118416

20. Zeng JR, Ning ZC, Wang YZ, et al. Implications of CD39 in immune-related diseases. Int Immunopharmacol. 2020;89(Pt A):107055. doi:10.1016/j.intimp.2020.107055

21. Enjyoji K, Kotani K, Thukral C, et al. Deletion of Cd39/Entpd1 results in hepatic insulin resistance. Diabetes. 2008;57(9)::23112320. doi:10.2337/db07-1265

22. Zhou R, Dang X, Sprague RS, et al. Alteration of purinergic signaling in diabetes: focus on vascular function. $J \mathrm{Mol}$ Cell Cardiol. 2020;140::1-9. doi:10.1016/j.yjmcc.2020.02.004

23. Kumar M, Lowery R, Kumar V. High-throughput screening assays for cancer immunotherapy targets: ectonucleotidases CD39 and CD73. SLAS Discov. 2020;25(3):320-326.

24. Martinez-Usatorre A, Godfroid C, Maroun CY, et al. Enhanced phenotype definition for precision isolation of precursor exhausted tumor-infiltrating CD8 T cells. Front Immunol. 2020;27(11):340. doi:10.3389/fimmu.2020.00340

25. Duarte MMF, Loro VL, Rocha JBT, et al. Enzymes that hydrolyze adenine nucleotides of patients with hypercholesterolemia and inflammatory processes. FEBS J. 2007;274(11):2707-2714. doi:10.1111/j.1742-4658.2007.05805.x

26. Meyer-Schwesinger C. The ins-and outs of podocyte lipid metabolism. Kidney Int. 2020;98(5):1087-1090. doi:10.1016/j. kint.2020.07.008

27. Ryu H, Kim J, Kim D, et al. Cellular and molecular links between autoimmunity and lipid metabolism. Mol Cells. 2019;42 (11):747-754.

International Journal of General Medicine

\section{Publish your work in this journal}

The International Journal of General Medicine is an international, peer-reviewed open-access journal that focuses on general and internal medicine, pathogenesis, epidemiology, diagnosis, monitoring and treatment protocols. The journal is characterized by the rapid reporting of reviews, original research and clinical studies

Submit your manuscript here: https://www.dovepress.com/international-journal-of-general-medicine-journal
28. Afshinnia F, Pennathur S. Lipids and cardiovascular risk with CKD. Clin J Am Soc Nephrol. 2020;15(1):5-7. doi:10.2215/CJN.13531119

29. Zhao RL, Qiao JJ, Zhang XM, et al. Toll-like receptor-mediated activation of CD39 internalization in BMDCs leads to extracellular ATP accumulation and facilitates P2X7 receptor activation. Front Immunol. 2019;10:2524. doi:10.3389/fimmu.2019.02524

30. Linden J, Koch-Nolte F, Dahl G. Purin release, metabolism and signaling in the inflammatory response. Annu Rev Immunol. 2019;37:325-347. doi:10.1146/annurev-immunol-051116-052406

31. Roberts VS, Cowan RJ, Alexander SI, et al. The role of adenosine receptors $\mathrm{A} 2 \mathrm{~A}$ and $\mathrm{A} 2 \mathrm{~B}$ signaling in renal fibrosis. Kidney Int. 2014;86(4)::685-692. doi:10.1038/ki.2014.244

32. Wang YM, McRae JL, Robson SC, et al. Regulatory T cells participate in CD39-mediated protection from renal injury. Eur J Immunol. 2012;42(9)::2441-2451. doi:10.1002/eji.201242434

33. Zhang X, Ouyang X, Xu ZJ, et al. CD8+CD103+ iTregs inhibit chronic graft-versus-host disease with lupus nephritis by the increased expression of CD39. Mol Ther. 2019;27(11):1-11. doi:10.1016/j.ymthe.2019.07.014

34. Roberts V, Lu B, Chia J, Cowan PJ, Dwyer KM. CD39 overexpression does not attenuate renal fibrosis in the unilateral ureteric obstructive model of chronic kidney disease. Purinergic Signal. 2016;12 (4)::653-660. doi:10.1007/s11302-016-9528-1

35. Fabbiano S, Menacho-Marquez M, Robles-Valero $J$, et al. Immunosuppression-independent role of regulatory $\mathrm{T}$ cells against hypertension-driven renal dysfuctions. Mol Cell Biol. 2015;35 (20):3528-3546. doi:10.1128/MCB.00518-15

36. Villa-Bellosta R. Synthesis of extracellular pyrophosphate increases in vascular smooth muscle cells during phosphate-induced calcification. Arterioscler Thromb Vasc Biol. 2018;38(9):2137-2147. doi:10.1161/ATVBAHA.118.311444

37. Dwyer KM, Kishore BK, Robson SC. Conversion of extracellular ATP into adenosine: a master switch in renal health and disease. Nat Rev Nephrol. 2020;16(9):509-524. doi:10.1038/s41581-020-0304-7

38. Silva AC, Morsch ALB, Zanin RF, et al. Enzymes that hydrolyze adenine nucleotides in chronic renal failure: relationship between hemostatic defects and renal failure severity. Biochim Biophys Acta. 2005;1741(2005):282-288. doi:10.1016/j.bbadis.2005.06.002

39. Friedman DJ, Rennke HG, Csizmadia E, et al. The vascular ectonucleotidase ENTPD1 is a novel renoprotective factor in diabetic nephropathy. Diabetes. 2007;56(9):2371-2379. doi:10.2337/db061593

40. Giorgi MD, Enjyoji K, Jiang G, et al. Complete deletion of Cd39 is atheroprotective in apolipoprotein E-deficient mice. J Lipid Res. 2017;58(7):1292-1305. doi:10.1194/jlr.M072132

41. Zhang Z, He JW, Fu WZ, et al. Calcification of joints and arteries: second report with novel NT5E mutations and expansion of the phenotype. J Hum Genet. 2015;60(10):561-564. doi:10.1038/ jhg.2015.85

across all disease areas. The manuscript management system is completely online and includes a very quick and fair peer-review system, which is all easy to use. Visit http://www.dovepress.com/ testimonials.php to read real quotes from published authors. 